\title{
Skin Cancer and Parkinson's Disease
}

\author{
Joaquim J. Ferreira, MD, PhD, ${ }^{1 *}$ Dulce Neutel, MD,${ }^{1}$ Tiago Mestre, MD, ${ }^{1}$ Miguel Coelho, MD, ${ }^{1}$ \\ Mário M. Rosa, MD, ${ }^{1}$ Olivier Rascol, $\mathrm{MD}, \mathrm{PhD},{ }^{2}$ and Cristina Sampaio, $\mathrm{MD}, \mathrm{PhD}^{1}$ \\ ${ }^{I}$ Neurological Clinic Research Unit, Institute of Molecular Medicine, Lisbon School of Medicine, Lisbon, Portugal \\ ${ }^{2}$ Departments of Clinical Pharmacology and Neurosciences, INSERM CIC-9302 and UMR-825, \\ University Hospital, Toulouse, France
}

\begin{abstract}
The report of an increased frequency of melanoma during the clinical development of rasagiline prompted a renewed interest in a possible association between skin cancer and Parkinson's disease (PD). The evaluation of this risk ended in a recommendation to perform a periodic dermatological examination as a follow-up measure of their treatment. The recognition of this safety concern lead to the need to clarify if the risk of skin cancer is indeed associated with PD and if levodopa or other anti-parkinsonian drugs might contribute to increase such risk. To answer these questions, we critically reviewed all clinical studies available concerning the association between skin cancer and PD. We found 26 studies on can-
\end{abstract}

cer occurrence in PD. The best data available suggest the risk of cancer is reduced in PD patients. However, specific cancers like thyroid and the female breast were reported at higher-thanexpected rates. Additionally, it was suggested that PD patients have a higher frequency of melanoma and non-melanoma skin cancers than the general population. The data on non-melanoma skin cancer are less robust than the data on melanoma. Causal factors remain unknown. Due to the weak association between skin cancer and PD, no robust recommendation can be made regarding the need for periodic dermatological screening. (C) 2010 Movement Disorder Society

Key words: Parkinson's disease; cancer; melanoma; skin
Concern about an increased risk of skin cancer in Parkinson's disease (PD) patients was first raised by Skibba (1972) based upon a case of recurrent malignant melanoma in a PD patient treated with levodopa (L-dopa). ${ }^{1}$ Since then, more than 50 cases of newly diagnosed melanoma, melanoma recurrence, or melanoma metastasis were reported in L-dopa-treated PD patients. $^{2}$

These case reports were sufficient to raise safety concerns to an extent that, since 1976, a formal contraindication exists for the use of L-dopa in PD patients with melanoma. For example, Sinemet and Madopar yield the warning " $(. .$.$) because levodopa may acti-$

*Correspondence to: Dr. Joaquim Ferreira, Centro de Estudos Egas Moniz, Faculdade de Medicina de Lisboa, 1649-028 Lisboa, Portugal.

E-mail: joaquimjferreira@net.sapo.pt

Potential conflict of interest: None reported.

Received 7 April 2008; Revised 28 September 2009; Accepted 29 September 2009

Published online 8 January 2010 in Wiley InterScience (www. interscience.wiley.com). DOI: 10.1002/mds.22855 vate a malignant melanoma, it should not be used in patients with suspicious, undiagnosed skin lesions or a history of melanoma." ${ }^{3}$ The association between levodopa therapy and melanoma was considered theoretically plausible because L-dopa is a substrate for the synthesis of dopamine and melanin. ${ }^{4-6}$

The finding of an increased frequency of melanomas during the clinical development of rasagiline prompted a renewed interest in a possible association of skin cancer and PD. The evaluation of this risk ended in a recommendation to perform periodic dermatological examinations in patients as follow-up measure of their treatment. ${ }^{7-9}$ A similar amendment was later added to the safety labels of pramipexole, ${ }^{10}$ ropinirole,$^{11}$ and selegiline. ${ }^{12}$

To review the association between skin cancers and PD, we critically analyzed the epidemiological and clinical studies available. First, we reviewed data on the global risk of cancer in PD. As a second step, we focused on specific studies evaluating the risk of melanoma and other skin cancers in PD patients. 


\section{SEARCH STRATEGY AND SELECTION CRITERIA}

We searched the database Medline (1966-2008) with the terms "cancer," "mortality," "melanoma," and "Parkinson's disease". We also searched referenced lists of identified studies on cancer and melanoma in PD and handsearched the abstract books of international congresses of movement disorders. All studies aimed at evaluating the frequency of any type of cancer or cancer related mortality in PD were selected.

Quality of published methodology data and related susceptibility to bias were assessed through a checklist approach for study design, diagnostic criteria of PD, type of cancer ascertainment, and statistical analysis.

\section{STUDIES ABOUT CANCER RISK IN PD}

The first comment about cancer in PD was made by Doshay in 1954 who concluded from the analysis of a case series that cancer was rare in "paralysis agitans." 13 Several other studies followed from other case series, chart reviews, prospective cohorts, or case-control studies. In Table 1, we present all known studies for which the primary or secondary objectives were to evaluate the frequency of cancer in PD patients.

Hoehn and Yahr investigated the cause of death in 194 patients with PD and found that 24 patients had died of malignant neoplasms. ${ }^{16}$ When compared with the expected number calculated using the New York population as reference (41 cases), they concluded that a lower rate of death by malignant neoplasm occurred in PD patients $(P<0.001) .{ }^{16}$ Interestingly, 5 cases of skin cancers from 69 cases of malignant neoplasms were reported. There was no description of the type of skin lesion and no comparison was made with the expected prevalence of skin cancers in the reference population.

Jansson and Jankovic ${ }^{19}$ retrospectively reviewed 406 medical charts of PD patients and identified 18 patients with cancer, when compared with an expected number of 41.9. The exception to these lower cancer rates were malignant melanoma ( 2 cases observed vs. 0.3 expected, $P=0.04)$ and thyroid tumors (3 cases, all in women, $P<0.001)$. Interestingly, non-melanoma skin cancers occurred less frequently than expected (10 cases observed vs. 49.9 expected, $P<0.0001)$. The L-dopa dose, duration of treatment, and other risk factors related to the disease were not included in the analysis. The authors concluded that the rate of cancer and non-mela- noma cases was low, but the number of cases of malignant melanoma was higher than expected.

Elbaz et al. $^{27}$ conducted a population-based casecontrol study to investigate the association of PD with nonfatal cancer. They used the medical records-linkage system of the Rochester Epidemiology Project (19761995). Each case was matched by age and sex to a general population control. The frequency of cancer in general was lower in PD cases (19.4\%) than in controls (23.5\%) (OR 0.79; 95\% CI 0.49-1.27). This pattern was more pronounced in women than in men and in patients aged 71 years or younger at onset of PD. They did not find an association between PD and nonfatal cancer. However, they did find a decreased prevalence of smoking-related cancers and an increased prevalence of malignant melanoma. This latter finding was based on 3 observed cases in the patient group.

The same authors ${ }^{28}$ used the same approach to investigate the risk of cancer after the diagnosis of PD. They included 196 patients and 185 control subjects in this study. The risk of cancer was higher among patients than in controls (RR 1.64; 95\% CI 1.15-2.35; $P=0.007)$. The increased risk was significant for non-melanoma skin cancers (RR 1.76; 95\% CI 1.07$2.89 ; P=0.03)$. Among PD patients, there was no relation between the risk of cancer and the cumulative dose of L-dopa or the use of other PD medications. No other types of cancer were found to be associated with PD. However, these analyses were hampered by the small sample size and a potential surveillance bias.

Moller et al. ${ }^{22}$ conducted a retrospective study in a cohort of 7,046 patients with a primary diagnosis of PD obtained from a Danish hospital discharge computerised register (during 1977-89). Information on cancer incidence and death among cohort members from their first recorded admission for PD until the end of 1990 was obtained from the Danish cancer registry and from the Danish registry of deaths (the average duration of follow-up was 4.6 years). The Danish Cancer Registry began reporting incidence data in 1943 and includes cases of non-melanoma skin cancers (basal cell and squamous cell carcinoma). The expected numbers of cancer cases were calculated from the person's years at risk among cohort members and the incidences of cancer in the Danish population. The overall incidence of cancer was lower than expected (relative risk $0.88,95 \%$ CI $0.8-1.0$ ). However, a significant increase in relative risk was seen for skin melanoma (relative risk 1.96, 95\% CI 1.1-3.2). Relative risks of other skin cancers also increased, although this was not statistically significant (relative risk 1.24, 95\% CI 1.0-1.5). 


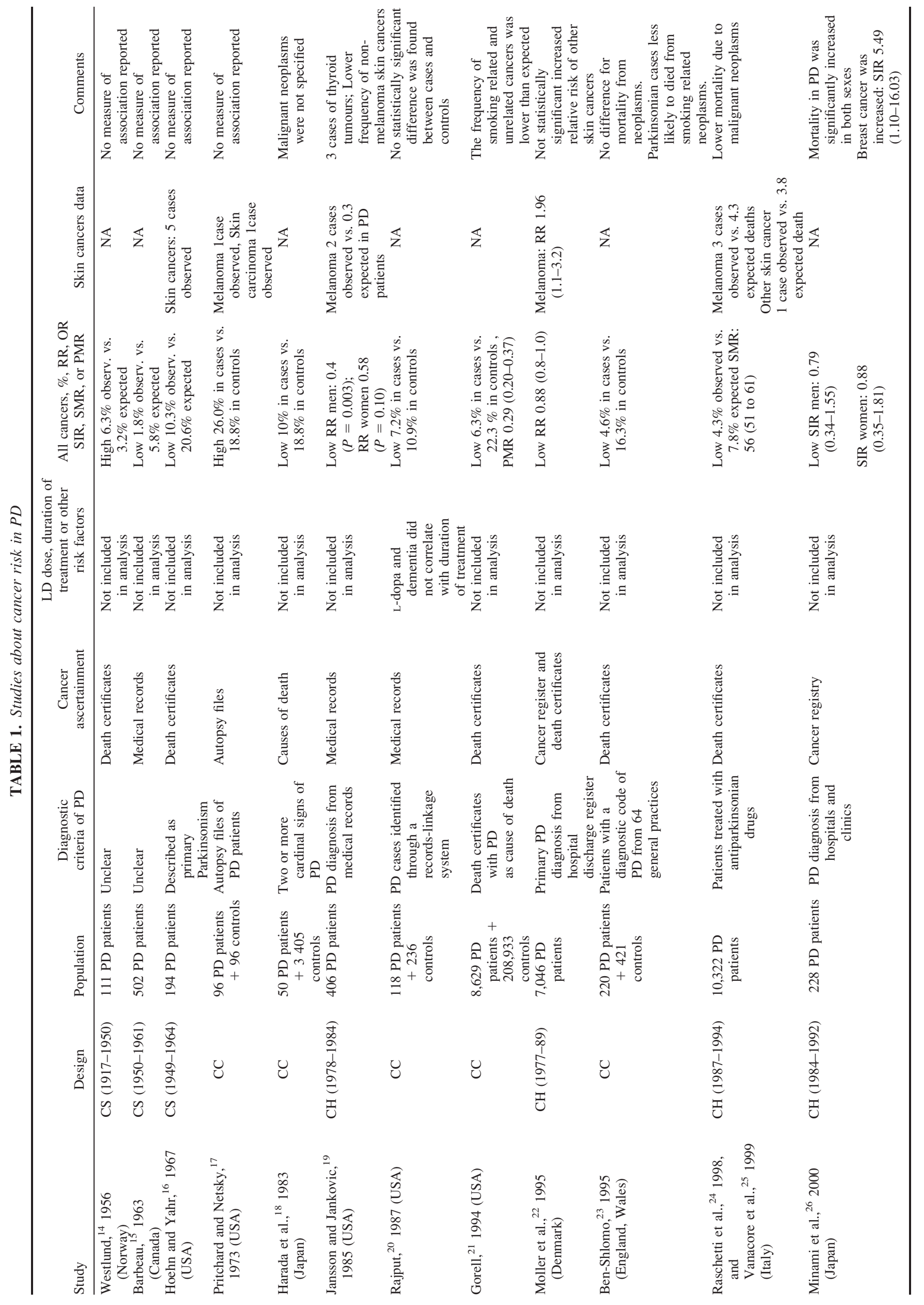




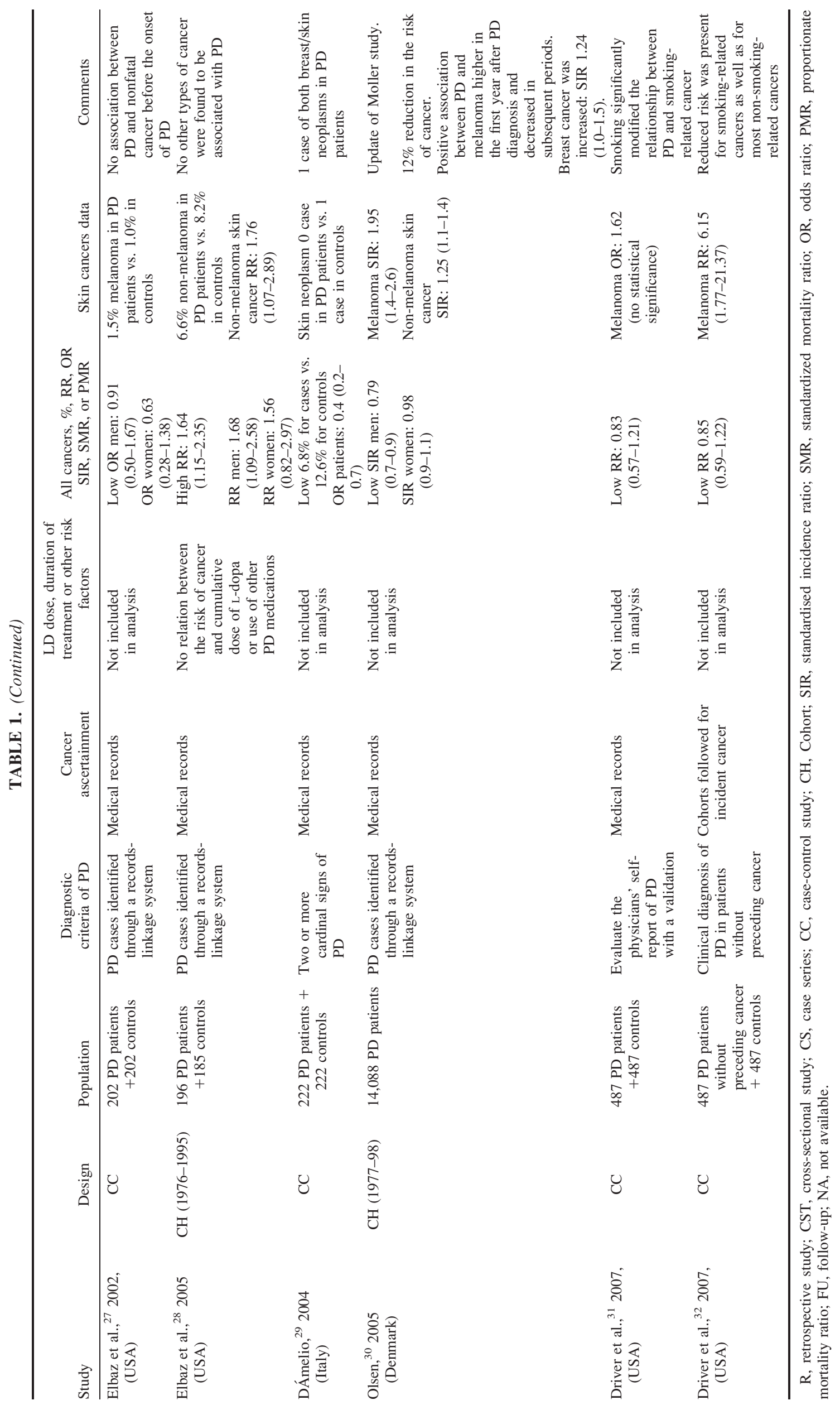


An update of the Moller study ${ }^{22}$ was recently published, covering a longer period (1977-98). ${ }^{30}$ Among 14,088 patients with a primary diagnosis of PD (average duration of follow-up of 5 years), 1,282 cancer cases were subsequently recorded compared to 1,464 expected cases, with a standardized incidence ratio (SIR) of 0.88 (95\% CI $0.8-1.0)$, that is equivalent to a $12 \%$ reduction in the risk of cancer. Significantly reduced risks were found for smoking-related cancers (e.g. lung (SIR, 0.38), larynx (SIR, 0.47), and urinary bladder (SIR, 0.52)) cancers. In contrast, increased risks were seen for malignant melanoma (SIR, 1.95; 95\% CI 1.4-2.6), non-melanoma skin cancer (1.25; 95\% CI 1.1-1.4), and breast cancer (1.24; 95\% CI 1.01.5). The association between $\mathrm{PD}$ and melanoma was higher in the 1st year after PD diagnosis and decreased in subsequent periods. As in the first study, there was also an increased relative risk for non-melanoma skin cancers, which reached statistical significance in the larger study. In both studies, there was no information on treatment and the suspected role of L-dopa as a risk factor for malignant melanoma could not be investigated.

Driver et al. ${ }^{31}$ conducted a nested case-control study within a prospective cohort of 22,071 US male physicians to estimate the association between the diagnosis of PD and the development of cancer. During the 22 years of follow-up, 487 cases of PD were identified and age-matched to 487 controls. The frequency of any cancer was lower in PD cases $(13.1 \%)$ than in controls $(14.8 \%)$. The same research group conducted another case-control study using the same cohort to evaluate cancer incidence following the diagnosis of PD. A total of 487 cases of PD without cancer were age-matched to reference participants who were alive and cancer-free at the time of PD diagnosis. A total of 121 cases of cancer were confirmed during a median follow-up of 5.2 years (PD) and 5.9 years (reference). Those with PD developed less cancer (11.0 versus $14.0 \%$ ), with an adjusted RR of 0.85 [95\% CI, 0.591.22]. Reduced risk was present for smoking-related cancers such as lung (RR, 0.32), colorectal (RR, 0.54), and bladder (RR, 0.68), as well as for most non-smoking-related cancers such as prostate cancer (RR, 0.74). In contrast, PD patients were at a significantly increased risk for melanoma (RR, 6.15; 95\% CI, 1.7721.37). ${ }^{32}$

The most robust evidence concerning the global risk of cancer in PD derives from the results of the Danish PD cohort studies. ${ }^{22,30}$ All other epidemiological studies have small samples and inadequate statistical power to conclusively assess the risk for skin cancer (mela- noma and non-melanoma) with reasonable precision. Overall, data available are consistent and sufficiently robust to conclude that $\mathrm{PD}$ is associated with a decreased risk for cancer when compared with the general population. However, some cancer types have been reported to occur in excess of expected numbers including malignant melanoma of the skin, other skin cancers, and cancers of the thyroid and the female breast.

\section{STUDIES ON SKIN CANCER RISK IN PD}

Recently, specific studies have investigated the problem of melanoma or other skin cancers in PD (Table 2). Different approaches were used to investigate the potential association: determination of the frequency of melanoma in PD patients' cohorts; to investigate the frequency of PD diagnosis in patients with a clinical history of melanoma; to investigate risk factors for the development of skin cancers in PD patients (e.g., Ldopa treatment, other anti-parkinsonian treatments, or other risk factors).

\section{FREQUENCY OF MELANOMA IN PD PATIENTS}

Olsen and coworkers ${ }^{33}$ conducted a population-based case-control study to investigate the prevalence of malignant melanoma, skin carcinoma, and other cancers before a first hospitalization or outpatient visit for PD. They identified 8,090 patients with a primary diagnosis of PD during the period of 1986-1998 from the National Danish Hospital Register. Each case was matched with four population controls selected at random from among inhabitants alive at the date of first hospital contact with the patient. The number of cancer cases since 1943 were obtained from the Danish Cancer Registry. The study found an increased prevalence of malignant melanoma and skin carcinoma before the first hospital contact for PD, with overall odds ratios of 1.44 (95\% CI 1.03-2.01) and 1.26 (95\% CI 1.11$1.43)$, respectively. Cancers showed a reduced prevalence at smoking-related sites.

A cross-sectional survey to assess the frequency and characteristics of skin neoplasms in PD patients was conducted in 12 medical centres in Israel. ${ }^{34}$ Of the 1,395 patients included, 9 patients $(0.6 \%)$ had a histologically confirmed diagnosis of malignant melanoma (1 invasive; 8 in situ), 14 patients $(1.0 \%)$ had melanoma in their medical history, and 6 patients had melanoma diagnoses before and 8 after their PD diagnosis. The total number of patients with current or prior mel- 


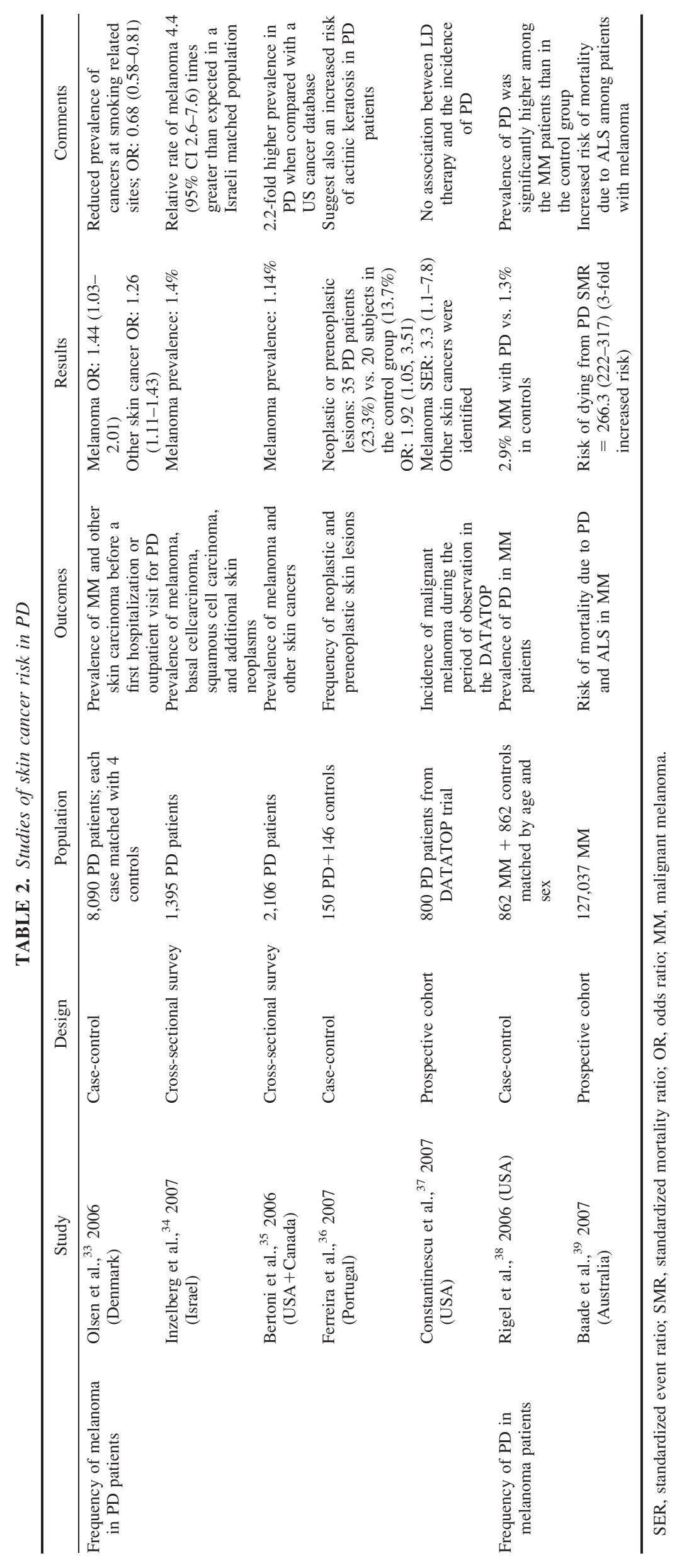


anoma was $20(1.4 \%)$. Occurrence of melanoma did not correlate with PD duration, $\mathrm{H} \& \mathrm{Y}$ stage, or L-dopa treatment. Analysis of prevalence data (5-year limited duration) for a comparable time period from the Israel National Cancer Registry suggested an overall relative rate of melanoma of 4.4 (95\% CI 2.6-7.6) times greater than expected based on an age- and sexmatched population. ${ }^{34}$

Bertoni et al. ${ }^{35}$ performed another cross-sectional survey in 2,106 North American PD patients who underwent a full-body dermatological examination and biopsy of any suspicious skin lesions. Of the 346 patients with suspicious pigmented lesions, 20 had histhologically confirmed in situ melanomas $(0.95 \%)$ and 4 had invasive melanomas $(0.19 \%)$. No relationship between the occurrence of melanoma (before or at examination) and L-dopa usage was observed. Prevalence (5-year limited-duration) of invasive melanoma in US PD patients $(\mathrm{n}=1,692)$ was 2.2 -fold higher $(95 \% \mathrm{CI}$ $1.21-4.17)$ than expected in age- and sex-matched populations in the National Surveillance Epidemiology and End Results-US Cancer Statistics Review database (SEER). Compared with American Academy of Dermatology screening programs, age- or sex-adjusted relative risk of any melanoma at screening was more than eight times higher for US patients.

A small cross-sectional survey found more neoplastic or pre-neoplastic lesions in PD patients $(23.3 \%)$ when compared to age matched controls $(13.7 \%)$ (OR 95\%CI $1.92[1.05,3.51]) .{ }^{36}$ Likewise, more cases of actinic keratosis $(19 \%)$ and basal cell carcinoma (3\%) were diagnosed in PD patients, suggesting that pre-neoplastic skin lesions, such as actinic keratosis, could also play a role in the increased risk of PD patients to develop melanoma.

Constantinescu et al. ${ }^{37}$ evaluated the frequency of malignant melanoma in the DATATOP clinical trial cohort. The DATATOP cohort included 800 patients enrolled between September 1987 and November 1988, and followed until 1994. Five cases of melanoma were found when compared with an expected number of 1.5 after adjusting for age and gender (standardized event ratio 3.3 [95\% CI 1.1-7.8]). Two cases of malignant melanoma were diagnosed before the L-dopa treatment onset, and 3 cases occurred after 1, 6, and 19 months. No conclusion could be made about an association between L-dopa therapy and the incidence of melanoma.

\section{FREQUENCY OF PD IN MELANOMA PATIENTS}

Rigel et al. ${ }^{38}$ performed a case-controlled study in 862 malignant melanoma patients and 862 controls with no history of melanoma or pigmented lesionrelated problems. Cases and controls were matched by age and gender. Cases of malignant melanoma were collected from US academic dermatology clinics.

Among the melanoma patients, 25 (2.9\%) cases had PD compared with 11 (1.3\%) controls with PD. The authors concluded that the odds of having PD was more than twofold greater in patients with malignant melanoma than in the control subjects.

Baade et al. ${ }^{39}$ conducted a cohort study of all patients diagnosed as having melanoma in Australia since 1982 $(\mathrm{n}=127,037)$. The subjects were followed through the end of 2001. Their cohort had a risk of death due to amyotrophic lateral sclerosis (ALS) that was 70\% higher (standardized mortality ratio $=169.4,95 \% \mathrm{CI}=127-$ 221) than the general population, and nearly a threefold increased risk of dying from PD (standardized mortality ratio $=266.3,95 \% \mathrm{CI}=222-317)$. These increased risks continued for long-term survivors, arguing against a surveillance effect (particularly for ALS).

\section{LEVODOPA AS A RISK FACTOR FOR MELANOMA}

Since the early 1970s, a number of case reports have suggested that L-dopa therapy increased the risk of cutaneous malignant melanoma. However, this safety concern was based on a limited number of anecdotal reports, and on cases where melanoma preceded Ldopa treatment, in which a formal causal-relationship evaluation would preclude such a link. There are also cases with no exacerbation or recurrence of melanoma in patients that were kept on L-dopa therapy. The data from these case reports are also limited in terms of patient characteristics that could be correlated with an increased risk for melanoma, such as sun exposure, family history, or fair skin.

The only epidemiological study that has specifically evaluated the role of L-dopa was conducted by Sober et al. ${ }^{40}$ They conducted a prospective survey in 1,099 patients from the Melanoma Clinical Cooperative Group. At the time of presentation of their primary melanoma, only 1 patient had been taking L-dopa. The authors concluded that L-dopa had no role in the induction of melanoma. No other formal epidemiological study has been conducted to test the hypothesis that $\mathrm{L}-$ dopa therapy for PD increases the risk of cutaneous malignant melanoma. Interestingly, the hypothesis that $\mathrm{L}-$ dopa could be toxic to the melanocytes was raised in the 1970s. ${ }^{41}$ Although its efficacy was never demonstrated, high doses of oral L-dopa were used in practice to treat metastatic malignant melanomas. ${ }^{42}$ 


\section{OTHER ANTI-PARKINSONIAN DRUGS AND SKIN CANCER}

There are no epidemiological studies evaluating the frequency of skin cancer in PD patients treated with any anti-Parkinsonian drug other than L-dopa. All data available are derived from adverse events reported in published clinical trials or registered in pharmacovigilance systems. ${ }^{43,44}$

\section{DISCUSSION}

From this analysis of all the data available regarding the association between PD and skin cancer, melanoma occurs at a higher frequency in PD patients when compared with the general population. Similarly, although not so robustly demonstrated, non-melanoma skin cancers appear at an increased frequency in PD patients. The studies available were not designed to enable conclusions to be made regarding the causal relationship between L-dopa and skin cancers.

Due to the heterogeneity of study designs and outcome measures, no statistical pooling of the results was appropriate to be conducted. Nevertheless, a descriptive analysis of the best data suggest a prevalence of melanoma in PD patients between 1.1 and $1.4 \%$ and a 1.5-3-fold increase in the incidence of melanoma. If we apply these estimates to the expected incidence of melanoma in the United States for subjects 65-years-old and older (65.4 per 100.000 per year; SEER 2001-2005), we presume an approximate incidence of 1 to 2 cases per 1000 PD patients per year (slight increased risk).

The theory linking L-dopa and melanoma was based on the shared biochemical pathways between the synthesis of both dopamine and melanin. The association between L-dopa and melanoma is therefore based on biological plausibility and a few case reports where data is too limited to determine the causality. ${ }^{1,45-47}$ In some cases, the short interval between the onset of Ldopa treatment and the diagnosis of melanoma makes it somewhat implausible that a carcinogenic effect is induced by L-dopa. On the other hand, the reported stronger association between PD and melanoma within the first years after PD diagnosis ${ }^{30}$ reduces the likelihood that these cases of melanoma are due to PD treatment and suggests the possibility of other pre-existing causal or confounding, unknown factors.

Additionally, the association of PD with non-melanoma skin cancers counters the theory of L-dopa as a causal factor, unless the biochemical pathway including L-dopa is common to all types of skin cancer. As one of the common causative factor is long-term sun exposure, ${ }^{48,49}$ it may be hypothesized that PD patients are more sensitive to sun-exposure-induced skin lesions. This may be due to a disease-specific susceptibility or to a photocarcinogenic potential of L-dopa or other anti-parkinsonian drugs. The increased rate of malignant melanoma and non-melanoma skin cancers before the diagnosis of PD weakens the hypothesis that skin cancers may be caused by the treatment of PD. ${ }^{33}$

Furthermore, although exogenous L-dopa was suggested to have some effect on melanin synthesis, consequently, stimulating melanogenesis and melanoma growth ${ }^{50}$ it has not been demonstrated that L-dopa is carcinogenic. Other theories for L-dopa-induced melanoma included inhibition of the pineal gland, ${ }^{51}$ increased plasma concentrations of growth hormone, ${ }^{52}$ and mediation of immunosuppression by enhancing secretion of melanocyte-stimulating hormone. ${ }^{51}$ Interestingly, L-dopa and other precursors in the biosynthetic pathway of melanin may have a toxic effect on melanoma in vitro. ${ }^{53}$ The two clinical surveys specifically conducted to evaluate the frequency of cutaneous lesions in PD patients concluded that an extremely high prevalence of melanoma existed $(1.4 \%$ in Israel and $1.1 \%$ in North America). However, the interpretation of these rates is difficult without a parallel control group or a valid external database. This is even more difficult knowing both that the incidence of melanoma has sharply increased in the last 70 years, ${ }^{54}$ and that the overall calculation of melanoma incidence in the general population is imprecise because rate figures are calculated based on data collection systems that cannot find cases of less-invasive disease.

The incidence of PD increases with age and the incidence of malignant melanoma has been increasing in recent decades. Consequently, it is expected that both PD and malignant melanoma will coincidentally affect several patients every year, even without a causal relationship between the two diseases. On the other hand, if the increased risk in PD patients is caused by environmental or genetic factors common to both diseases, it would be expected that the association between PD and skin cancer be bidirectional (i.e., that the risk for malignant melanoma would also be increased before a diagnosis of PD) that cannot be excluded with the data available. In the scenario of an independent common cause for PD and melanoma, we would expect either to have melanoma first and PD after or vice-versa. Nevertheless, given that cancer has a much higher mortality it might happen that there is no time to develop PD once cancer occurs. In this situation, it would result that it should be more frequent to identify PD 
cases were cancer was found after the neurological diagnosis, than the inverse.

So far all the melanoma reports refer to cutaneous melanoma, with no data regarding the occurrence of ocular melanoma. This may be because of its rarity or be justified by its being more difficult to screen in primary dermatologic surveys.

In summary, from the data available, there is:

1. Consistent data supporting an association between cutaneous melanoma and PD;

2. A possible association between non-melanoma skin cancers and PD;

3. Insufficient data to conclude on the association between L-dopa and melanoma in PD patients;

4. Insufficient data to conclude on the association between rasagiline, selegiline, ropinirole, pramipexole or other anti-parkinsonian drugs and melanoma or other skin cancers in PD patients;

5. Insufficient data about the risk factors for skin cancer in PD patients.

\section{IMPLICATIONS FOR PRACTICE}

Physicians and PD patients should be made aware of the association between PD and skin cancers.

No robust recommendation can be made regarding the need for periodic dermatological screening.

\section{IMPLICATIONS FOR RESEARCH}

In our opinion, there is a clear need to understand the risk factors for the association between melanoma and other non-melanoma cancers and PD. To investigate the putative role of medications requires a prospective, casecontrol study designed to examine the main risk factors for the development of skin cancers in PD patients. This study should be a large study focused on patients with more than 5 years of disease treatment and without any restriction in terms of anti-parkinsonian drugs. It will be crucial to have parallel prospective controls using the same case-ascertainment methods.

Any clinical survey or pharmacoepidemiological study about this safety problem should also include a balanced effort to identify not only melanoma but also all neoplastic and pre-neoplastic skin lesions.

Financial Disclosure: In the past 12 months, the authors have the following information to disclose. Joaquim Ferreira, Consultancies: TEVA, Lundbeck, Ipsen, Solstice, Novartis, GlaxoSmithKline, Solvay, Grunenthal, BIAL; Advisory Boards: GlaxoSmithKline, Novartis, TEVA, Lundbeck, Allergan, Solvay, BIAL. Mário Rosa, Honoraria: Tecnifar, Grunenthal. Olivier Rascol: Consultancies: Boehringer Ingelheim, Eisai, GlaxosmithKline, Novartis, Schering, Solvay, Teva
Neuroscience, Lundbeck and UCB; Grants: Boehringer Ingelheim, Eisai, GlaxosmithKline, Novartis, Solvay, Teva Neuroscience and Lundbeck. Cristina Sampaio, Consultancies: In all cases the fees / honoraria due are paid to department and not received personally: Lundbeck, Abbott, Bial, Boeringher -LMS Group Schering-Plough, Solvay.

Author Roles: Joaquim Ferreira conceived and designed the review; collected, analyzed and interpreted the data; drafted the article; review and critique of manuscript; concepted, organised, and execution of the research project; designed, executed, and review and critique of statistical analysis. Dulce Neutel collected, analyzed and interpreted the data; help draft the article; organization and execution of research project; review and critique of statistical analysis and manuscript. Tiago Mestre, Miguel Coelho and Mário M. Rosa helped draft the article, analyzed and interpreted the data and critically revised it; organization and execution of the research project; review and critique of statistical analysis and manuscript. Olivier Rascol and Cristina Sampaio analyzed and interpreted the data; critically revised the article; conception of research project; and review and critique of statistical analysis.

\section{REFERENCES}

1. Skibba JL, Pinckley J, Gilbert EF, Johnson RO. Multiple primary melanoma following administration of levodopa. Arch Pathol 1972;93:556-561.

2. Pfutzner W, Przybilla B. Malignant melanoma and levodopa: is there a relationship? Two new cases and a review of the literature. J Am Acad Dermatol 1997;37:332-336.

3. Medical Economics Company, Inc. Physicians' desk reference. 57th ed. Montvale, NJ: Medical Economics Company, Inc., 2003. p 1111.

4. Kincannon J, Boutzale C. The physiology of pigmented nevi. Pediatrics 1999;104:1042-1045.

5. Siple JF, Schneider DC, Wanlass WA, Rosenblatt BK. Levodopa therapy and the risk of malignant melanoma. Ann Pharmacother 2000;34:382-385.

6. Fiala KH, Whetteckey J, Manyam BV. Malignant melanoma and levodopa in Parkinson's disease: causality or coincidence? Parkinsonism Relat Disord 2003;9:321-327.

7. Parkinson Study Group. A randomized placebo-controlled trial of rasagiline in levodopa-treated patients with Parkinson disease and motor fluctuations: the PRESTO study. Arch Neurol 2005;62: 241-248.

8. Parkinson Study Group. A controlled trial of rasagiline in early Parkinson disease: the TEMPO Study. Arch Neurol 2002;59: 1937-1943.

9. Teva Pharmaceutical Industries Ltd. Azilect ${ }^{\circledR}$ package insert. Kfar Saba, Israel: Teva Pharmaceutical Industries Ltd; 2006.

10. Boehringer Ingelheim International $\mathrm{GmbH}$. Mirapex ${ }^{\circledR}$ package insert. Ridgefield, CT: Boehringer Ingelheim International $\mathrm{GmbH}$; 2006.

11. GlaxoSmithKline. Requip ${ }^{\mathbb{R}}$ package insert. Research Triangle Park, NC: GlaxoSmithKline; 2005.

12. Valeant Pharmaceuticals International. Zelapar ${ }^{\circledR}$ package insert. Costa Mesa, CA: Valeant Pharmaceuticals International; 2006.

13. Doshay LJ. Problem situations in the treatment of paralysis agitans. J Am Med Assoc 1954;156:680-684.

14. Westlund KHA. Cancer as a cause of death among patients with other chronic disease. JAMA 1956;162:1003.

15. Barbeau A JJ-G. Parkinson et cancer. L'Union Med du Canada 1963;92:169-174. 
16. Hoehn MM, Yahr MD. Parkinsonism: onset, progression and mortality. Neurology 1967;17:427-442.

17. Pritchard PB, III, Netsky MG. Prevalence of neoplasms and causes of death in paralysis agitans. A necropsy study. Neurology 1973;23:215-222.

18. Harada H, Nishikawa S, Takahashi K. Epidemiology of Parkinson's disease in a Japanese city. Arch Neurol 1983;40.

19. Jansson B, Jankovic J. Low cancer rates among patients with Parkinson's disease. Ann Neurol 1985;17:505-509.

20. Rajput AH, Offord KP, Beard CM, Kurland LT. A case-control study of smoking habits, dementia, and other illnesses in idiopathic Parkinson's disease. Neurology 1987;37:226-232.

21. Gorell JM JCRB. Parkinson's disease and its comorbid disorders: an analysis of Michigan mortality data, 1970-1990. Neurology 1994;44:1865-1868.

22. Moller H, Mellemkjaer L, McLaughlin JK, Olsen JH. Occurrence of different cancers in patients with Parkinson's disease. BMJ 1995;310:1500-1501.

23. Ben-Shlomo YMM. Survival and cause of death in a cohort of patients with parkinsonism: possible clues to aetiology? J Neurol Neurosurg Psychiatry 1995;58:293-299.

24. Raschetti R, Spila-Alegiani S, Vanacore N, Ancona C, Meco G. Mortality in a population-based cohort of patients treated with antiparkinsonian drugs. Acta Neurol Scand 1998;97:20-26.

25. Vanacore N, Spila-Alegiani S, Raschetti R, Meco G. Mortality cancer risk in parkinsonian patients: a population-based study. Neurology 1999;52:395-398.

26. Minami Y, Yamamoto R, Nishikouri M, Fukao A, Hisamichi S. Mortality and cancer incidence in patients with Parkinson's disease. J Neurol 2000;247:429-434.

27. Elbaz A, Peterson BJ, Yang P, et al. Nonfatal cancer preceding Parkinson's disease: a case-control study. Epidemiology 2002;13: 157-164.

28. Elbaz A, Peterson BJ, Bower JH, et al. Risk of cancer after the diagnosis of Parkinson's disease: a historical cohort study. Mov Disord 2005;20:719-725.

29. D'Amelio M, Ragonese P, Morgante L, et al. Tumor diagnosis preceding Parkinson's disease: a case-control study. Mov Disord 2004; 19:807-811.

30. Olsen JH, Friis S, Frederiksen K, McLaughlin JK, Mellemkjaer L, Moller H. Atypical cancer pattern in patients with Parkinson's disease. Br J Cancer 2005;92:201-205.

31. Driver JA, Kurth T, Buring JE, Gaziano JM, Logroscino G. Prospective case-control study of nonfatal cancer preceding the diagnosis of Parkinson's disease. Cancer Causes Control 2007;18: 705-711.

32. Driver JA, Logroscino G, Buring JE, Gaziano JM, Kurth T. A prospective cohort study of cancer incidence following the diagnosis of Parkinson's disease. Cancer Epidemiol Biomarkers Prev 2007;16:1260-1265.

33. Olsen JH, Friis S, Frederiksen K. Malignant melanoma and other types of cancer preceding Parkinson disease. Epidemiology 2006; 17:582-587.

34. Inzelberg R, Rabey JM, Djaldetti R, et al. Elevated prevalence of malignant melanoma in Israeli patients with Parkinson's disease. Mov Disord 2006;22:S169.
35. Bertoni JM, Arlette JP, Fernandez HH, et al. Parkinson's disease and melanoma: an epidemiologic evaluation. Ann Neurol 2006; 60 (Suppl 3):S71-S72.

36. Ferreira J, Silva JM, Freire R, et al. Skin cancers and precancerous lesions in Parkinson's disease patients. Mov Disord 2007;22: 1471-1475.

37. Constantinescu R, Romer M, Kieburtz K. Malignant melanoma in early Parkinson's disease: the DATATOP trial. Mov Disord 2007;22:720-722.

38. Rigel DS, Patel Z, Bolognia J, Eichler C, Ellis DL, Friedman RJ. Evaluation of Parkinson's disease (PD) prevalence in patients with malignant melanoma. Mov Disord 2006;21:S58.

39. Baade PD, Fritschi L, Freedman DM. Mortality due to amyotrophic lateral sclerosis and Parkinson's disease among melanoma patients. Neuroepidemiology 2007;28:16-20.

40. Sober AJ, Wick MM. Levodopa therapy and malignant melanoma. JAMA 1978;240:554-555.

41. Wick MM, Byers L, Frei E, III. L-dopa: selective toxicity for melanoma cells in vitro. Science 1977;197:468-469.

42. Gurney H, Coates A, Kefford R. The use of L-dopa and carbidopa in metastatic malignant melanoma. J Invest Dermatol 1991; 96:85-87.

43. Parkinson Study Group. A randomized placebo-controlled trial of rasagiline in levodopa-treated patients with Parkinson disease and motor fluctuations: the PRESTO study. Arch Neurol 2005;62: 241-248.

44. Parkinson Study Group. A controlled trial of rasagiline in early Parkinson disease: the TEMPO Study. Arch Neurol 2002;59: 1937-1943.

45. Lieberman AN, Shupack JL. Levodopa and melanoma. Neurology 1974;24:340-343.

46. Kochar AS. Development of malignant melanoma after levodopa therapy for Parkinson's disease. Report of a case and review of the literature. Am J Med 1985;79:119-121.

47. Weiner WJ, Singer C, Sanchez-Ramos JR, Goldenberg JN. Levodopa, melanoma, and Parkinson's disease. Neurology 1993;43: 674-677.

48. Whiteman DC, Stickley M, Watt P, Hughes MC, Davis MB, Green AC. Anatomic site, sun exposure, and risk of cutaneous melanoma. J Clin Oncol 2006;24:3172-3177.

49. Ivry GB, Ogle CA, Shim EK. Role of sun exposure in melanoma. Dermatol Surg 2006;32:481-492.

50. Weiner WJ, Singer C, Sanchez-Ramos JR, Goldenberg JN. Levodopa, melanoma, and Parkinson's disease. Neurology 1993;43: 674-677.

51. Sandyk R. Accelerated growth of malignant melanoma by levodopa in Parkinson's disease and role of the pineal gland. Int $\mathrm{J}$ Neurosci 1992;63:137-140.

52. Boyd AE, III, Lebovitz HE, Pfeiffer JB. Stimulation of humangrowth-hormone secretion by L-dopa. N Engl J Med 1970;283: $1425-1429$.

53. Rampen FH. Levodopa and melanoma: three cases and review of literature. J Neurol Neurosurg Psychiatry 1985;48:585-588.

54. Rigel DS, Carucci JA. Malignant melanoma: prevention, early detection, and treatment in the 21 st century. CA Cancer J Clin 2000;50:215-236. 